\title{
Parametric analysis of gravity offshore structures: Part 1
}

\author{
JAIME DOMINGUEZ \\ Escuela Tecnica Superior de Ingenieros Industriales de Sevilla, Avda. Reina Mercedes, Serilla, \\ Spain \\ and ENRIQUE ALARCON \\ ETS Ingenieros Industriales, Universidad Politecnica, Madrid 3, Spain
}

\section{INTRODUCTION}

The objective of this paper is to analyse the influence of the variation of some parameters used in the analysis of the dynamic response of offshore structures under the action of wind generated waves.

The structural response has been obtained by stochastic methods using two discretization models. One with lumped parameters, using translational degrees of freedom (d.o.f.) and the other with one-dimensional finite elements. Using each of these methods the problem has been solved with several d.o.f., analysing the influence of the number of d.o.f. on the results.

\section{SOLUTION METHOD}

To calculate the response by stochastic methods, the linearized equation of motion minimizing the error in the least square sense has been used ${ }^{1}$ :

$$
\left[M_{T}\right]\{\ddot{u}\}+[\mathbf{C}]\{\dot{u}\}+[K]\{u\}=\left\{P_{I}\right\}+\left\{P_{A}\right\}=\{P\}
$$

where $\left[M_{T}\right]$ is the mass matrix including the structural mass and the added mass introduced by the water; $[\mathbf{C}]$ is the damping matrix formed by the structural and the hydrodynamic damping; $[K]$ is the stiffness matrix; $\left\{P_{I}\right\}$ and $\left\{P_{A}\right\}$ are the inertia and drag force vectors respectively and $\{u\}$ is the nodal displacement vector.

If the discretization has been made using lumped parameters and considering only translational degrees of freedom the terms of the aforementioned matrices can be written:

$$
\begin{gathered}
M_{T j j}=M_{j j}+\left(K_{m}-1\right) \rho V_{j} \\
\mathbf{C}_{i j}= \begin{cases}C_{i j}+K_{d} \rho \frac{A j}{2} \sigma_{\dot{r} j} \sqrt{8 / \pi} & i=j \\
C_{i j} & i \neq j\end{cases} \\
P_{l j}=K_{m} \rho V_{j} \ddot{v}_{j} \\
P_{A j}=K_{d} \rho-\frac{A_{j}}{2} \sigma_{i j} \sqrt{8 / \pi} \dot{v}_{j}
\end{gathered}
$$

The terms of $[K]$ can be calculated by any of the known methods. Usually it is done applying static condensation to another matrix with translation and rotation degrees of freedom.

In equations (2) to (5), $K_{m}$ and $K_{d}$ are the inertia and drag coefficients respectively; $M_{j j}$ is the structural mass at node $j ; \rho$ is the water density; $V_{j}$ is the volume of the structure lumped at $j ; C_{i j}$ is the structural damping coefficient; $A_{j}$ is the structural projected area in direction of flow; $\sigma_{i j}$ is the standard deviation of the relative velocities of water and structure; and $\dot{v}_{j}$ is the water velocity at node $j$.

If the discretization is done using one-dimensional finite elements, the terms of the structural element matrices can be written:

$$
\begin{gathered}
M_{T i j}=\int_{0}^{l} m(x) g_{i}(x) g_{j}(x) \mathrm{d} x+\int_{0}^{d}\left(K_{m}-1\right) \rho V(x) g_{i}(x) g_{j}(x) \mathrm{d} x \\
\mathbf{C}_{i j}=C_{i j}+\int_{0}^{d} \sqrt{8 / \pi} K_{d} \rho \frac{A(x)}{2} \sigma_{r}(x) g_{i}(x) g_{j}(x) \mathrm{d} x \\
P_{i}=P_{I i}+P_{A i}=\int_{0}^{d} K_{m} \rho V(x) g_{i}(x) \ddot{v}(x) \mathrm{d} x+\int_{0}^{d} \sqrt{8 / \pi} K_{d} \rho \times \\
\frac{A(x)}{2} \sigma_{\dot{r}}(x) g_{i}(x) \mathrm{d} x
\end{gathered}
$$

where $g(x)$ are the shape functions of the structural elements, $l$ their total length and the integrals between 0 and $d$ extend along the submerged parts of the structural elements.

To obtain some of the terms of the equations (7) and (8), it is necessary to know $\sigma_{\dot{r}}$ as a function of the various points of the structure. Thus, during the iterative process ${ }^{1}$ required to calculate $\sigma_{i}$, it will be necessary to know the structural displacements at all points $(u(x))$, which can be obtained from the shape functions and the nodal displacements.

Applying the nodal superposition method, equation (1) can be written in normal coordinates:

$$
\left[M^{*}\right]\{\ddot{y}\}+\left[C^{\prime}\right]\{\dot{y}\}+\left[K^{*}\right]\{y\}=\left\{P^{*}\right\}
$$




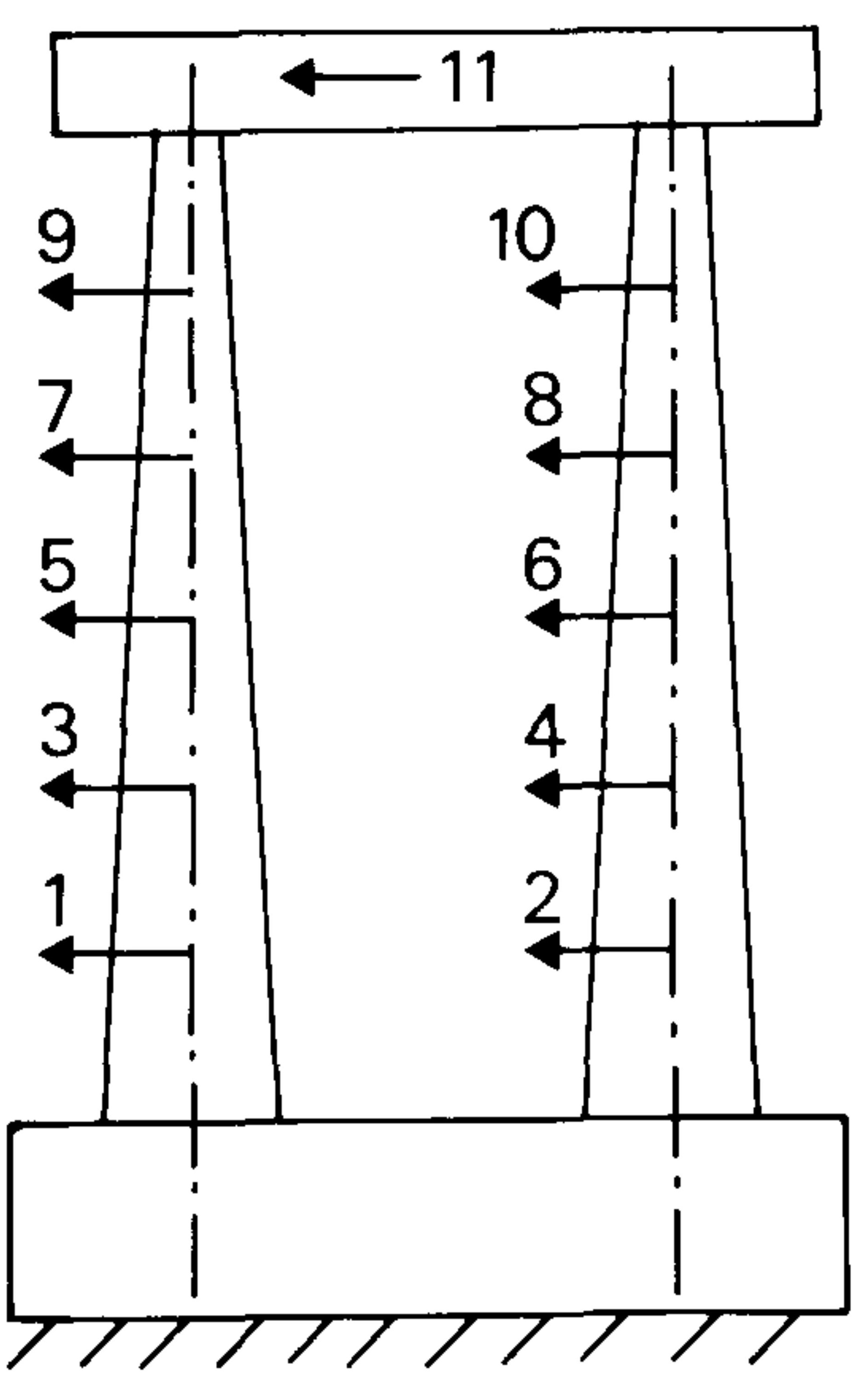

a

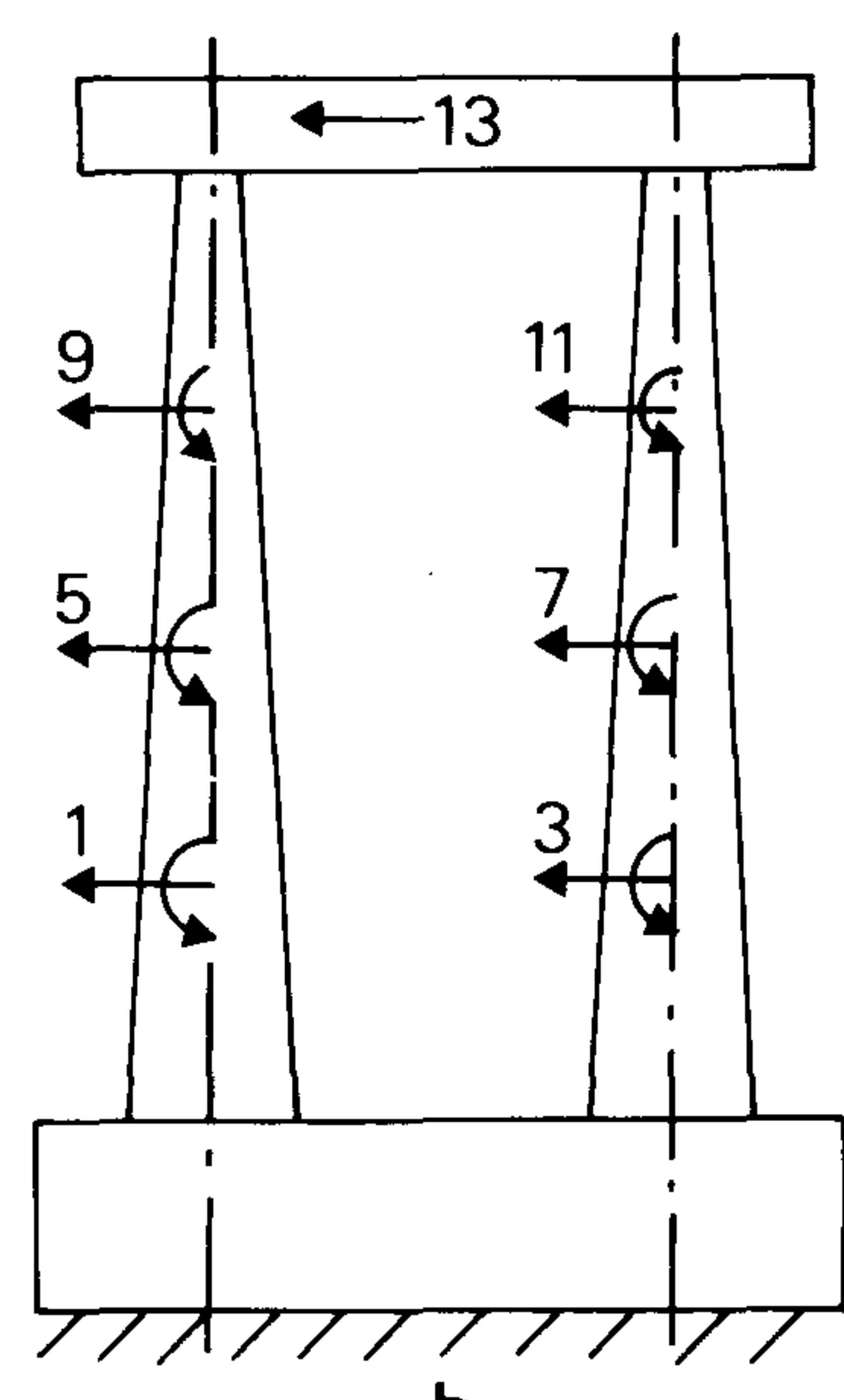

b
Figure 1. Two models for discretization

In this equation, the damping matrix is not diagonal due to the hydrodynamic damping. However, to avoid a cumbersome formulation $\left[C^{\prime}\right]$ can be approximated by diagonal matrix $\left[C^{*}\right]$, minimizing the mean square error using the same method ${ }^{1}$. After it, the new equation is:

$$
\left[M^{*}\right]\{\ddot{y}\}+\left[C^{*}\right]\{\dot{y}\}+\left[K^{*}\right]\{y\}=\left\{P^{*}\right\}
$$

Solving equation (10) by stochastic methods, the displacement spectral density function in normal coordinates can be written:

$$
S_{y_{j, l_{k}} l_{k}}(\omega)=H_{j}^{*}(\omega) H_{k}(\omega) S_{P_{j}^{*} P_{k}^{*}}(\omega)=H_{j}^{*}(\omega) H_{k}(\omega) \sum_{l} \sum_{m} \varphi_{l j} \varphi_{m k} S_{p_{i} P_{m}}(\omega)
$$

where $H_{k}(\omega)$ and $H_{j}^{*}(\omega)$ are the complex frequency response function and its complex conjugate for modes $k$ and $j$ repsectively; $\varphi_{l j}$ and $\varphi_{m k}$ are terms of the modal matrix and $S_{p_{i} p_{m}}$ is the force cross-spectral density function of the degrees of freedom $l$ and $m$ which can be calculated from the sea spectrum.

Taking into account the transformation:

$$
\{u\}=[\varphi]\{y\}
$$

the response spectral density in global coordinates is:

$$
S_{u_{p} u_{q}}=\sum_{j} \sum_{k} \varphi_{p j} \varphi_{q k} S_{y_{j} y_{k}}(\omega)
$$

From equations (11) and (13) and considering the wave process and the structural response as a zero mean Gaussian ergodic random process, a stochastic analysis of the response may be carried out ${ }^{2.3}$.

\section{RESULTS}

For the analysis of results depending on the number of degrees of freedom and the discretization method used for modelling the system, the response of a gravity offshore structure with 4 columns in $150 \mathrm{~m}$ water depth has been studied. Since the structure is symmetrical and a wave propagating in the direction of the axis of symmetry is considered, only half of the structure has been studied. As we are going to study the difference in results but not the absolute values of the response some simplifications which will equally affect both models have been made. The base and the deck of the structure are considered to be rigid and no difference in phase is taken into account for loads acting on both columns.

The problem has been solved using lumped parameter models with 5, 7.9,11,13 and 15 d.o.f. and shape function models with 5, 9 and 13 d.o.f. In Fig. 1, two of them are shown one with 11 d.o.f. discretized using the first method and another with 13 d.o.f. discretized using the second one.

Eace case has been solved taking for $K_{m}$ and $K_{d}$ the values of 1.8 and 0.5 respectively ${ }^{4}$. First, natural frequencies of each model were computed. In Fig. 2 the values of the frequencies for the 1st, 2nd, 3rd modes in each model are shown and compared with those obtained for the 13 d.o.f. discretization using one-dimensional finite elements. The calculated values of the 13 d.o.f. modal frequencies are $2.14,7.98$ and $8.72 \mathrm{rad} / \mathrm{sec}$ respectively. The Figure shows that the difference between values obtained for the first three modes using 9 or more d.o.f. is never greater than $2.5 \%$

In a similar way a comparison has been made between the most probable extreme-values obtained at the deck, considering a fully developed sea with a power spectral density function proposed by Pierson-Moskowitz ${ }^{5}$ and produced by a wind speed of $30 \mathrm{~m} / \mathrm{sec}$. A comparison of the computed values is showed in Fig. 3. In that case, the reference value is the response of the same model used before having a value of $27.7 \mathrm{~cm}$. It can be verified that the difference with the reference value of the response of models with 9 or more d.o.f. is never greater than $3 \%$.

In order to study the influence of the wave regime on different models, the response of three of them to other regimes has been obtained. For this purpose the 5 and 11
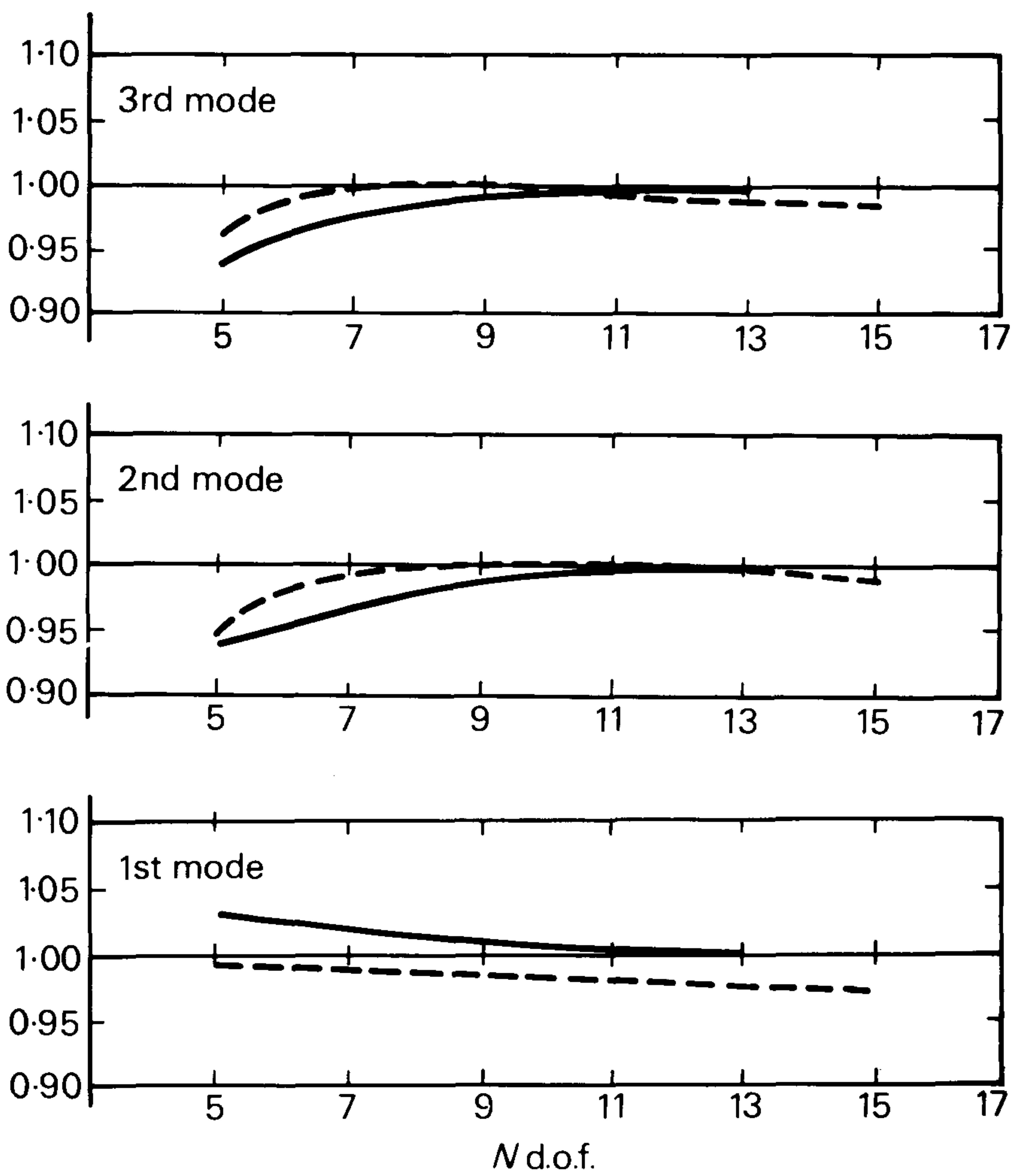

Figure 2. Comparison of natural frequencies of platform. -_, One-dimensional finite element; -..., lumped parameter 


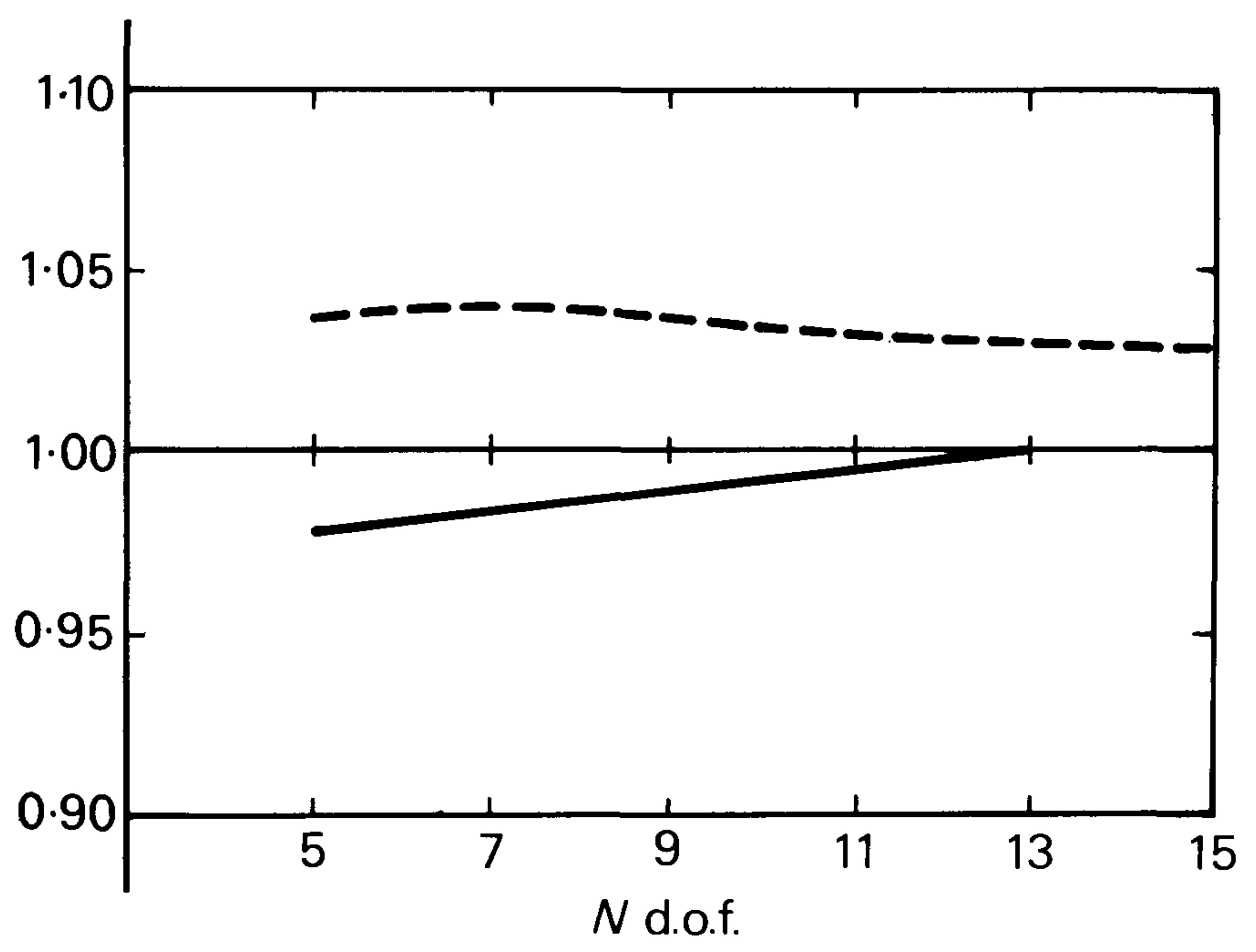

Figure 3. Comparison of deck response of platform.---, One-dimensional finite element; -..--, lumped parameter

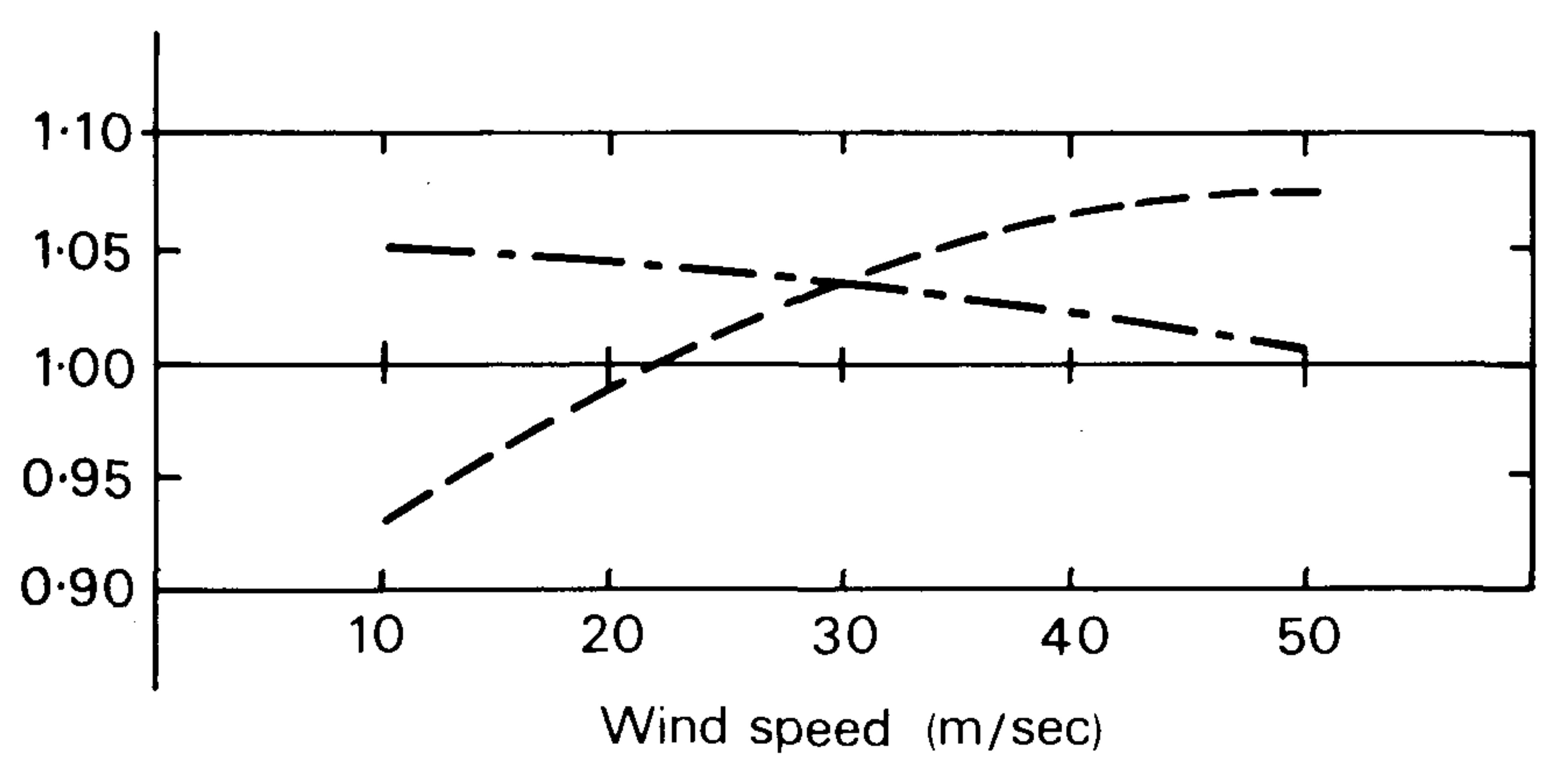

Figure 4. Comparison of response of platform to P.M. spectrum at different wind speeds. -, $13 ;--\ldots,-11$; $\ldots, 5$ d.o.f.

d.o.f. lumped parameter models and the system with 13 d.o.f. discretized by the shape functions method have been used for comparing their response to fully developed seas generated by wind velocities ranging from 10 to $50 \mathrm{~m} / \mathrm{sec}$.

Figure 4 shows the deck response evolution in the aforementioned systems when the wind velocity changes within the specified limits. The values represented are the relations between the response of the 5 and 11 d.o.f. models and those obtained for the 13 d.o.f. case. The difference between the responses of 11 and 13 d.o.f. systems is never higher than $5 \%$ and decreases for higher sea states becoming almost zero for $50 \mathrm{~m} / \mathrm{sec}$.

To ascertain the reason for these differences, the deck displacement spectral density for the three models for different wind speeds has been analysed. The spectral densities are almost equal for 11 and 13 d.o.f. except near the resonance peak. The 5 d.o.f. system presents higher differences than the 11 d.o.f. one at all frequencies. Figure 5 shows the spectral densities for the 10 and $30 \mathrm{~m} / \mathrm{sec}$ wind speeds.

The reason why the response in the 11 d.o.f. system at resonance is higher than the 13 d.o.f. one is the difference between their natural frequencies. Thus, as the 11 d.o.f. system natural frequency is a little lower, its response to waves with frequencies lower than the natural frequency will be a little higher than in the 13 d.o.f. model.

In Fig. 5(a) it can be seen that the influence of the resonance peak in the response is higher for lower wind speeds; i.e. waves have dominant frequencies nearer the natural frequencies of the models. Thus the difference between their response is higher for low wind speeds.

Simultaneously to the calculation of response, the accuracy achieved in the iterations was checked. In this way, it has been verified that only small improvements are obtained in the resulting accuracy during iteration. Even for wave regimes with a high value of KeuleganCarpenter number, differences between variances of velocities and displacements obtained in the first step, and those of the second step are usually less than $1 \%$; and between second and third step differences are less than $0.1 \%$.

\section{CONCLUSIONS}

According to the previous discussion, it can be said that in structures of the kind considered sufficient accuracy can be obtained through analysis with a relatively small number of d.o.f

In the selection of this number, attention should be paid to the fact that only a slight improvement of accuracy is reached when increasing the number of d.o.f. over a certain value with the disadvantage of more than linear increase of computer time and storage capacity.

With the discretization methods, it can be said that both are accurate enough with a few d.o.f. and any of them
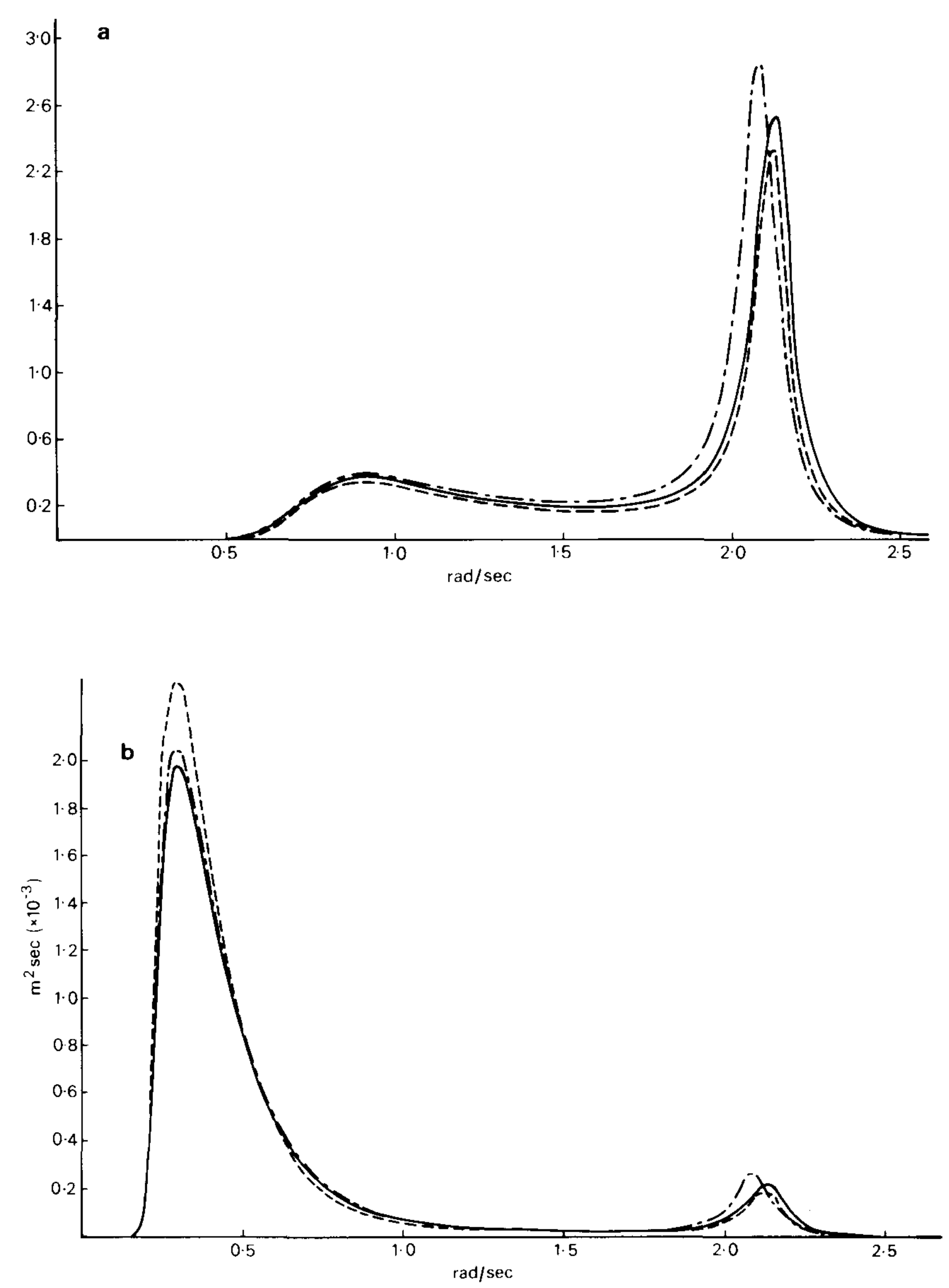

Figure 5. Spectral densities of response of platform. $\ldots, 5 ;-\ldots \ldots$ d.o.f. (a) Wind speed $=10 \mathrm{~m} / \mathrm{sec} ;$ (b) wind speed $=30 \mathrm{~m} / \mathrm{sec}$ 
can be used successfully to solve the response problem. Furthermore the difference in computer time between the two methods is small and almost constant for any number of d.o.f.

Finally, it can be said that for this kind of structure where the Keulegan-Carpenter number $K c \ll 15$, it is possible to obtain sufficient accuracy without the iteration process.

However, it is not possible to say the same for steel structures because in that case the Keulegan-Carpenter number is higher and drag forces become more important. Thus when the iterative process is not performed the error should be analysed depending on the Keulegan-. Carpenter number.

\section{REFERENCES}

1 Malhotra, A. K. and Penzien, J. Nondeterministic analysis of offshore structures, J. Eng. Mech. Dir. (ASCE) Dec. 1970, 96, 985

2 Cartwright, D. E. and Longuet-Higgins, M. S. The statistical distributions of the maxima of a random function, Proc. R. Soc. $(A)$ 1956, 237, 212

3 Davenport, A. G. Note on the distribution of the larguest value of a random function with application to gust loading, Proc. Inst. Civil Eng. 1964, 28, 187

4 Hogben, N., Miller, B. L., Searle, J. W. and Ward, G. Estimation of fluid loading on offshore structures, Proc. Inst. Civil Eng. Sept. 1977, 63, 515

5 Pierson, W. J. and Moskowitz, L. A proposed spectral form for fully developed wind seas based on the similarity theory of S. A. Kitaigorodskii, J. Geophys. Res. 1964, 69, 5181 